\title{
COMPLETE HYPERSURFACES IN CYLINDERS
}

\author{
THOMAS HASANIS
}

(Received 25 March 1987; revised 12 February 1988)

Communicated by K. Mackenzie

\begin{abstract}
We consider the extent of certain complete hypersurfaces of Euclidean space. We prove that every complete hypersurface in $E^{n+1}$ with sectional curvature bounded below and non-positive scalar curvature has at least $(n-1)$ unbounded coordinate functions.

1980 Mathematics subject classification (Amer. Math. Soc.): primary 53 C 40; secondary 53 B 20.

Keywords and phrases: complete hypersurface, bounded sectional curvature, scalar curvature.
\end{abstract}

In this note we consider complete hypersurfaces of Euclidean space that lie entirely within the convex body bounded by certain "cylindrical" hypersurfaces. The results are interesting because they supply information concerning the extent of certain complete hypersurfaces of Euclidean space. Results in this direction have already appeared in [1].

We take a fixed splitting of Euclidean space $E^{n+1}=E^{n-k+1} \times E^{k}$. The cylindrical hypersurface $S_{\lambda}^{n-k} \times E^{k}$ of $E^{n+1}$, where $S_{\lambda}^{n-k}$ denotes the hypersphere in the subspace $E^{n-k+1}$, with center at the origin and radius $\lambda$, bounds a convex body that we denote by $C(n, \lambda, k)$. Note that $C(n, \lambda, 0)$ is the closed ball of radius $\lambda$ in $E^{n+1}$.

First we prove the following theorem.

THEOREM 1. Let $M^{n}$ be a complete, oriented, $n$-dimensional $(n \geq 2)$ hypersurface in $E^{n+1}$, with sectional curvature bounded below, assume $M^{n}$ is contained

(C) 1989 Australian Mathematical Society 0263-6115/89 \$A2.00+0.00 
in a $C(n, \lambda, k)$, with $0 \leq k \leq n-2$. Then

$$
\sup R \geq \frac{(n-k)(n-k-1)}{\lambda^{2}},
$$

where $R$ stands for the scalar curvature of $M^{n}$.

The following lemma, whose proof is an easy consequence of the law of inertia for quadratic forms, will be used in the proof of the theorem.

LEMMA 1. Let $V$ be an $n$-dimensional Euclidean space and $W$ be an $m$ dimensional subspace of $V$. Assume $B$ is a symmetric bilinear form on $V$ with corresponding self-adjoint linear map $A$. If for some constant $\alpha>0$ and any nonzero $X \in W$ we have $B(X, X)>\alpha|X|^{2}$, then $A$ has at least $m$ eigenvalues greater than $\alpha$.

PROOF OF THEOREM 1. Let $\alpha_{1}, \ldots, \alpha_{k}$ be an orthonormal basis in the subspace $E^{k}$. We consider the smooth function

$$
f=\frac{1}{2}\left(\langle x, x\rangle-\sum_{i=1}^{k}\left\langle x, a_{i}\right\rangle^{2}\right),
$$

where $x$ is the position vector for the points of $M$ and $\langle$,$\rangle stands for the usual$ inner product in $E^{n+1}$. It is obvious that $f$ is well defined on $M$, independently of the origin we choose on the fixed subspace $E^{k}$, and $f \leq \lambda^{2} / 2$, since $M$ lies in $C(n, \lambda, k)$.

Using the Gauss formula, we obtain easily for the gradient and hessian of $f$ that

$$
\begin{gathered}
\langle X, \operatorname{grad} f\rangle=\left\langle X, x-\sum_{i=1}^{k}\left\langle x, a_{i}\right\rangle a_{i}\right\rangle, \\
\nabla^{2} f(X, X)=|X|^{2}-\sum_{i=1}^{k}\left\langle X, a_{i}\right\rangle^{2}+\langle L X, X\rangle\left\langle N, x-\sum_{i=1}^{k}\left\langle x, a_{i}\right\rangle a_{i}\right\rangle,
\end{gathered}
$$

for every tangent vector $X$, where $N$ is a chosen unit normal to $M$, and $L$ stands for the Weingarten map of $M$. By setting $y=x-\sum_{i=1}^{k}\left\langle x, a_{i}\right\rangle a_{i}$, we get

$$
f=\frac{1}{2}|y|^{2}, \quad \operatorname{grad} f=y_{T}
$$

and

$$
\nabla^{2} f(X, X)=|X|^{2}-\sum_{i=1}^{k}\left\langle X, a_{i}\right\rangle^{2}+\langle L X, X\rangle\langle N, y\rangle,
$$

where $y_{T}$ is the component of $y$ tangent to $M$. 
Applying Omori's theorem [3], we conclude that there exists a sequence $\left\{P_{m}\right\}$ of points of $M$ such that

$$
\begin{gathered}
\lim _{m \rightarrow \infty} f\left(P_{m}\right)=\sup _{M} f \\
\operatorname{lgrad} f\left|\left(P_{m}\right)=\right| y_{T} \mid\left(P_{m}\right)<\frac{1}{m}
\end{gathered}
$$

and

$$
\nabla^{2} f(X, X)=|X|^{2}-\sum_{i=1}^{k}\left\langle X, a_{i}\right\rangle^{2}+\langle L X, X\rangle\langle N, y\rangle<\frac{1}{m}|X|^{2},
$$

for any nonzero tangent vector $X$ at $P_{m}$. Let $\sup _{M} f=\frac{1}{2} \lambda_{0}^{2}$; then $0<\lambda_{0} \leq \lambda$. Since $|y|^{2}=\left|y_{T}\right|^{2}+\langle y, N\rangle^{2}$, we get from (1) and (2) that $\lim _{m \rightarrow \infty}|\langle N, y\rangle|\left(P_{m}\right)=$ $\lambda_{0}$. From the last relation we conclude that, taking a constant positive number $\varepsilon<\lambda_{0}$, there exists a natural number $m_{0}$ such that for $m \geq m_{0}$ we have

$$
\lambda_{0}-\varepsilon \leq|\langle y, N\rangle|\left(P_{m}\right) \leq \lambda_{0} .
$$

Now, the subspace $W_{m}=\left(T_{P_{m}} M\right) \cap\left(E^{k}\right)^{\perp}$ of $T_{P_{m}} M$ has dimension $\operatorname{dim} W_{m} \geq$ $n-k$. Thus for any nonzero $X \in W_{m}$, we get from (3)

$$
|X|^{2}+\langle L X, X\rangle\langle N, y\rangle<\frac{1}{m}|X|^{2}
$$

or

$$
|\langle L X, X\rangle|>\frac{\left(1-\frac{1}{m}\right)}{\lambda_{0}}|X|^{2} .
$$

Without loss of generality, we can choose a subsequence of $\left\{P_{m}\right\}$, which we denote also by $\left\{P_{m}\right\}$, such that at $P_{m}$ we have

$$
\langle L X, X\rangle>\frac{\left(1-\frac{1}{m}\right)}{\lambda_{0}}|X|^{2}, \quad \text { for any nonzero } X \in W_{m},
$$

and thus from (3) and (4) we conclude that

$$
-\lambda_{0} \leq\langle y, N\rangle\left(P_{m}\right) \leq-\left(\lambda_{0}-\varepsilon\right) .
$$

Now from (3) and (6) at $P_{m}$ and for any nonzero $X \in T_{P_{m}} M$ we obtain

$$
\frac{\langle L X, X\rangle}{|X|^{2}}>\frac{1-\sum_{i=1}^{k}\left\langle\frac{X}{|X|}, a_{i}\right\rangle^{2}-\frac{1}{m}}{-\langle y, N\rangle} .
$$

For the principal curvatures of our hypersurface we adopt the numbering $k_{1} \geq$ $\cdots \geq k_{n}$ with corresponding eigenvectors $E_{1}, \ldots, E_{n}$. Then, since $k_{i}\left(P_{m}\right)=$ $\left\langle L E_{i}, E_{i}\right\rangle /\left|E_{i}\right|^{2}$, we get from (7) that

$$
\lim \inf k_{i}\left(P_{m}\right) \geq 0, \quad \text { for all } i=1, \ldots, n \text {. }
$$


Now, Lemma 1 and (5) imply that at any $P_{m}$ there are at least $n-k$ principal curvatures, for example $k_{1}, \ldots, k_{n-k}$, greater than $(1-1 / m) / \lambda_{0}$. Because $n-k \geq$ 2 , we can assume that

$$
\lim \sup k_{i}\left(P_{m}\right)<\infty, \quad \text { for all } i=1, \ldots, n ;
$$

otherwise for the sectional curvature $K$ we would have $\lim \sup K=\infty$ and then $\sup R=\infty$, since the sectional curvature is bounded below, which makes the assertion trivial. By using (8) and (9) we obtain

$$
\lim \inf k_{i} k_{j}\left(P_{m}\right) \geq 0, \quad \text { for all } i, j=1, \ldots, n \text {. }
$$

Finally at $P_{m}$ we have

$$
\begin{aligned}
R\left(P_{m}\right) & =\sum_{\substack{i, j=1 \\
i \neq j}}^{n-k} k_{i} k_{j}\left(P_{m}\right)+2 \sum_{i=1}^{n} \sum_{\substack{j=n-k+1 \\
i<j}}^{n} k_{i} k_{j}\left(P_{m}\right) \\
& >(n-k)(n-k-1) \frac{\left(1-\frac{1}{m}\right)^{2}}{\lambda_{0}^{2}}+2 \sum_{i=1}^{n} \sum_{\substack{j=n-k+1 \\
i<j}}^{n} k_{i} k_{j}\left(P_{m}\right),
\end{aligned}
$$

and using (10), we have

$$
\sup R \geq \limsup R \geq \frac{(n-k)(n-k-1)}{\lambda_{0}^{2}},
$$

or, since $\lambda_{0} \leq \lambda, \sup R \geq(n-k)(n-k-1) / \lambda^{2}$, which completes the proof of the theorem.

Easy consequences of Theorem 1 are the following two corollaries which generalize results due to Leung [2].

COROLLARY 1. Let $M^{n}$ be a complete, oriented, hypersurface in $E^{n+1}$ with sectional curvature bounded below. Assume $M^{n}$ has non-positive scalar curvature; then $M^{n}$ has at least $(n-1)$ unbounded coordinate functions. In particular, $M^{n}$ is unbounded in $E^{n+1}$.

PrOOF. We assume that $M^{n}$ has exactly $k$ unbounded, and thus $(n-k)$ bounded, coordinate functions. Then $M^{n}$ is contained in a $C(n, \lambda, k)$. If $k \leq$ $n-2$, then by Theorem 1 we obtain that $\sup R>0$, which is a contradiction. Thus $k \geq n-1$.

COROLLARY 2. Let $M^{n}$ be a complete, oriented, hypersurface in $E^{n+1}$ with sectional curvature bounded below. Assume $M^{n}$ is contained in a closed ball of radius $\lambda$; then

$$
\sup R \geq \frac{n(n-1)}{\lambda^{2}}
$$


Theorem 1 does not cover two interesting cases $(k=n$ or $n-1)$. In fact, the proof of Theorem 1 breaks down when $M^{n}$ is between two parallel hyperplanes $(k=n)$ or when $M^{n}$ is in the interior of $S_{\lambda}^{1} \times E^{n-1}(k=n-1)$. Can Theorem 1 be true in these two cases?

By making an additional assumption, we can also cover these two cases.

THEOREM 2. Let $M^{n}$ be a complete, oriented, $n$-dimensional $(n \geq 2)$ hypersurface in $E^{n+1}$, whose scalar curvature $R$ is bounded below and whose mean curvature $H$ satisfies $|H| \leq H_{0}$, where $H_{0}$ is a positive constant. Suppose $M^{n}$ is in a $C(n, \lambda, k)$, with $0 \leq k \leq n$; then

$$
\sup R \geq \frac{(n-k)(n-k-1)}{\lambda^{2}} .
$$

The proof of this theorem is based on the following

LEMMA 2. Let $M^{n}$ be as in Theorem 2. Then either $M^{n}$ intersects every hyperplane of $E^{n+1}$, or $\sup R \geq 0$.

ProOF. Suppose $M^{n}$ has no intersection with a hyperplane $\Pi$ of $E^{n+1}$. So $M^{n}$ is in a half-space $W$ determined by $\Pi$. We choose an origin $O \in W$ and let $e$ be the unit normal to $\Pi$ which points in the other half-space. Now, we decompose $e$ at every point $P \in M^{n}$ in a component $e_{T}$ tangent to $M^{n}$, and a component normal to $M^{n}$ :

$$
e=e_{T}+\langle e, N\rangle N
$$

where $N$ is a chosen unit normal to $M^{n}$.

The height function $g=\langle x, e\rangle$ is bounded above. For the gradient and hessian of $g$ we obtain at every $P \in M$ that

$$
\begin{gathered}
\operatorname{grad} g=e_{T}, \\
\nabla^{2} g(X, X)=\langle e, N\rangle\langle L X, X\rangle,
\end{gathered}
$$

where $X \in T_{P} M$ and $L$ is the Weingarten map with respect to $N$. The choice of $O$ and $e$ implies sup $g \leq d(O, \Pi)$, where $d$ is the euclidean distance in $E^{n+1}$.

From the Gauss equation, by simple algebra, using our hypotheses, we deduce that the sectorial curvature of $M^{n}$ is bounded in absolute value. Therefore we may apply Omori's theorem to the function $g$. So there exists a sequence $\left\{P_{m}\right\}$ of points of $M$ such that

$$
\begin{gathered}
\lim _{m \rightarrow \infty} g\left(P_{m}\right)=\sup g, \\
|\operatorname{grad} g|\left(P_{m}\right)=\left|e_{T}\left(P_{m}\right)\right| \leq \frac{1}{m}
\end{gathered}
$$


and

$$
\nabla^{2} g(X, X)=\langle e, N\rangle\langle L X, X\rangle<\frac{1}{m}|X|^{2},
$$

for any nonzero $X \in T_{P_{m}} M$.

Since $|e|=1$, by using (11) and (15) we get $1-\langle e, N\rangle^{2}<1 / m^{2}$, at each $P_{m}$, or

$$
\sqrt{1-1 / m^{2}}<|\langle e, N\rangle|
$$

From (17), without loss of generality we may assume that there exists a subsequence of $\left\{P_{m}\right\}$, which we denote also by $\left\{P_{m}\right\}$, so that at each $P_{m}$ we have

$$
\sqrt{1-1 / m^{2}}<-\langle e, N\rangle \leq 1 \text {. }
$$

Moreover, from (16), (18) for $X=E_{i}(i=1, \ldots, n)$, the eigenvectors of $L$ at $P_{m}$, we get $k_{i}\left(P_{m}\right)>-1 / \sqrt{m^{2}-1}$, for all $i$, and hence

$$
\liminf k_{i}\left(P_{m}\right) \geq 0, \quad \text { for all } i \text {. }
$$

Now, the boundedness of $R$ and $H$ imply

$$
\lim \sup k_{i}\left(P_{m}\right)<\infty, \text { for all } i,
$$

and thus, by using (19), we conclude that $\lim \inf k_{i} k_{j}\left(P_{m}\right) \geq 0$ and so $\sup R \geq 0$.

Proof of TheOREM 2. This is a special case of Theorem 1 for $k \leq n-2$, and an immediate consequence of Lemma 2 for $k=n$ or $n-1$.

An immediate consequence of Theorem 2 is the following

COROLLARY 3. Let $M^{n}$ be a complete, oriented minimal hypersurface in $E^{n+1}$, with scalar curvature bounded below. Then either $\sup R=0$, or $M^{n}$ has, in any coordinate system, $n+1$ unbounded coordinate functions.

\section{References}

[1] Th. Hasanis and D. Koutroufiotis, 'Immersions of Riemannian manifolds into cylinders', Arch. Math. 40 (1983), 82-85.

[2] P.-F. Leung, 'Complete hypersurfaces on non-positive Ricci curvature', Bull. Austral. Math. Soc. 27 (1983), 215-219.

[3] H. Omori, 'Isometric immersions of Riemannian manifolds', J. Math. Soc. Japan 19 (1967), 205-214.

\section{Department of Mathematics \\ University of Ioannina 45110 \\ Ioannina}

Greece 